Volume 9. No. 7, July 2021

International Journal of Emerging Trends in Engineering Research

Available Online at http://www.warse.org/IJETER/static/pdf/file/ijeter09972021.pdf

https://doi.org/10.30534/ijeter/2021/09972021

\title{
A Survey on Pneumonia Detection Methods Using Computer-aided Diagnosis
}

\author{
Agrima Jain $^{1}$, Neha Mehra ${ }^{2}$, Preeti Khare ${ }^{3}$ \\ ${ }^{1}$ Department of Computer Engineering, S.G.S.I.T.S, Indore, India, jainagrima11@gmail.com \\ ${ }^{2}$ Department of Computer Engineering, S.G.S.I.T.S, Indore, India, mehra.neha40@ gmail.com \\ ${ }^{3}$ Department of Computer Engineering, S.G.S.I.T.S, Indore, India, preetikhr4@ gmail.com
}

\begin{abstract}
Recent advances in computer-assisted identification support the diagnosis of Pneumonia using imaging. Therefore, there are many activities available for diagnosing pneumonia using Computer-Aided Diagnosis. This paper provides research into the in-depth study strategies used to diagnose pneumonia. The principle intention of this paper is to introduce the study of pneumonia, to visualize the practice of current work in this field, to recognize the leftover issues and future patterns in this field. Pneumonia is numbered eighth among the main ten reasons for death in the US. As per WHO, "consistently it murders around 1.4 million kids younger than five" this is an exceptionally treatable illness. There is subsequently a requirement for investigation and improvement on PC helped tests with the goal that the chances of pneumonia are decreased and patients are treated before they arrive at a deadly region.
\end{abstract}

Key words : Chest X-ray Images, Computer-aided Diagnostics, Deep Learning, Pneumonia.

\section{INTRODUCTION}

Pneumonia is a sickness that influences the lungs. It very well may be brought about by Bacteria, Viruses, or Fungi. It is a hazardous infection. In this infection, the air sacs become loaded up with discharge and different liquids. Pneumonia causes around the 700,000 kid to pass every year and influences 7 percent of the total populace. Pneumonia is recorded as one of the primary drivers of death in the United States. Consistently 3.7 lakh youngsters pass on in India, of which half of the youngsters die from pneumonia. Patients with pneumonia may have side effects like coughing that produces phlegm or sometimes blood, fever, windedness or trouble breathing, chill or shuddering, weariness, perspiring, chest or muscle torment. Those most in danger of having pneumonia are small kids or individuals beyond 65 years old. Individuals with existing medical conditions are likewise in more danger. Hazard factors and conditions that may build the danger of pneumonia include: fever, openness to different synthetics, smoking or weighty drinking, ongoing medical procedure, late wounds, long clinic stay or escalated care, affliction like emphysema, HIV/AIDS, or different illnesses of the lungs influencing the resistant framework. Now and again pneumonia can reach major issues, like respiratory disappointment, the spread of infection, the liquid around the lungs, uncontrolled irritation all through the body. This situation can also be very dangerous, so it is important to take medical help immediately if you experience these symptoms. This disease is often overlooked and untreated to the point of death, especially in older medical patients. It is the cause of death in youngsters under five years. Pneumonia can affect children and families everywhere. It can be stopped for children with certain interventions and treated at minimum cost, with medication and little care. For all these reasons there is a need for research and development on computer-assisted diagnostics to reduce pneumonia.

Pneumonia can be determined with a Chest X-ray, CT scan, Chest ultrasound, A needle biopsy of the lungs, and chest MRI. Recently, X-ray images of the chest are one of the way to diagnose pneumonia. X-ray plays an important role in epidemiological studies and clinical care. X-rays are the most prevalent screening method that is more widely available and preferred over CT scans because CT scans can take longer. $\mathrm{X}$-ray imaging and advanced CT scans can not be available at most retrospective sites. Another issue with Pneumonia is that the elements which describe the presence of the infection are now and again blended in with different sicknesses so it is hard for radiologists to analyze this illness. Deep learning strategies are used to solve all these problems. Biomedical diagnostics uses Deep learning techniques for image classification to obtain an exact finding of the illness. In this paper, we are surveying the ways to detect pneumonia using Computer-aided Diagnosis.

\subsection{Types of Pneumonia}

Bacterial pneumonia: It happens when the body is debilitated by disease, ailing health, mature age, or contamination, and microscopic organisms into the lungs. This pneumonia can 
Agrima Jain et al., International Journal of Emerging Trends in Engineering Research, 9(7), July 2021, 890 - 895

influence all ages and is particularly perilous if you have been mishandling liquor, smoking, smoking cigarettes, going through a medical procedure, having respiratory disease, or having a weak immune system. Treatment of bacterial pneumonia is finished utilizing anti-infection treatment.

Viral pneumonia: Viruses, common cold, are all cases of pneumonia. Viral pneumonia will improve its own.

Mycoplasma pneumonia: It affects all ages. It has various signs and symptoms and is also called atypical pneumonia.

Other pneumonia: They can be caused by other infections including fungi.

\subsection{Groups having the highest risk of Pneumonia}

- Children younger than five years,

- People who smoke,

- People having certain medical conditions,

- Adults ages 65 and older.

\subsection{Symptoms of Pneumonia}

The different types of symptomatic pneumonia Infectious include blue lips and nails, fever, cough, heavy sweating, rapid breathing, wheezing, severe chest pain with deep breath or cough, cough. Symptoms of viral pneumonia are similar to bacterial pneumonia which can be followed by headaches, pneumonia, muscle aches, weakness, whooping cough, and mycoplasma pneumonia have a variety of symptoms, including a severe cough that can produce sputum.

\section{RELATED WORK}

Much work has already be done in pneumonia detection field by using Computer-aided Diagnosis and the latest improvements in Computer-aided Diagnosis methods allow them to be used in various fields [1-5]. Various writers have effectively proposed distinctive biomedical image recognition methods like skin cancer classification, hemorrhage identification, arrhythmia identification, and diabetic retinopathy discovery. The future and challenges of medical image processing have been discussed by M.I.Razaak [6]. A Deep Learning model for dermatologist-level characterization of skin cancer has been presented by Andre [7] and A strategy for the portrayal of prostrate in MRI volumes utilizing $\mathrm{CNN}$ was proposed by F.Milletari [8]. A strategy for distinguishing diabetic retinopathy in retinal supports photos was proposed by Varun [9], A method of deep learning for brain hemorrhage detection in CT scans used by Grewal [10] and Y. Bar examined chest pathology detection by methods dependent on deep learning[11]. Two deep three-dimensional (3D) customized mixed link network (CMixNet) models for lung knob detection and classification were used by Nasrullah et al. [12]. Yao L et al. combined long- short term memory network(LSTM) and DenseNet to find out the dependencies between abnormalities [13]. Some authors have been also worked on pneumonia classification. Earth mover's Distance (EMD) to distinguish contaminated

pneumonia lungs from ordinary non-tainted lungs was proposed by Khatri et al [14]. CNN model for pneumonia classification is used by Rahib et al. [15] and Okeke et al. [ 16]. Cohen et al. [17] and Rajaraman et al. [18] shown assuring results. Rajaraman et al. explain the performance of customized CNN to distinguish pneumonia and furthermore attempted to separate among bacterial and viral sorts in pediatric CXRs. The Region-based convolutional neural network was used by Sirazitdinov et al. [19] for portioning the pulmonary pictures with picture expansion for pneumonia identification. To obtain an area under the curve without any pre-training Lakhani and Sundaram used the AlexNet and GoogLeNet neural network with data augmentation [20]. To detect 14 different pathologies, including pneumonia, Rajpurkar et al. used cheXNeXt, a deep CNN of 121 layers [21]. Pre-trained DenseNet -121 was used to identify 14 thoracic diseases. For pneumonia diagnosis and classification Saraiva et al. [22], Ayan et al. [23] and Rahman et al. [24] used deep learning-based methods. Based on chest computed tomography (CT) images. A novel multi-scale heterogeneous three-dimensional (3D) convolutional neural network (MSH-CNN) was proposed by Xiao et al. [25]. For pneumonia detection, a hierarchical CNN structure and a novel loss function, sin loss is used by $\mathrm{Xu}$ et al. [26]. For pneumonia identification, both global and local features for image segmentation Jaiswal et al. use [27]. 3D deep CNN which had shortcut connections used by Jung et al. [28]. Vikash et al. used different neural networks and combined them and reached the final prediction using majority voting only Vikash combine prediction of the different neural networks [28]. Mohd. Farukh Hashmi, Satyarth katihar, Avinash G Keskar, Neeraj Dhanraj Bokde, and Zong Woo Geem integrates five deep learning models by using a weighted classifier that [30]. The weight for each model is based on each model's performance on the testing dataset. The main contribution of this paper is to survey the pneumonia detection techniques using deep learning.

\section{METHODS USED TO DETECT PNEUMONIA}

- Because Blood tests: By doing this test we are supposed to identify that the infection is present and if yes, then how much it has spread to the bloodstream. The amount of oxygen in your bloodstream can be tested by Arterial blood gas testing.

- Chest X-ray: X-Ray: images of bones, lungs, organs, and internal tissues are used to diagnose pneumonia. 
Agrima Jain et al., International Journal of Emerging Trends in Engineering Research, 9(7), July 2021, 890 - 895

- Pulse Oximetry: In this test, we measure the presence of oxygen in the blood. Here a little machine called an oximeter is utilized to gauge the presence of oxygen in the blood. This test is easy in this test a sensor is tapped onto a finger and when the machine turned on, a red light can be found in the sensor.

- Sputum culture: This test is done on the cough that comes up from the lungs and into the mouth. It's often used to see if there's an infection in the lungs.

- Chest CT scan: This is a strategy that uses a blend of $\mathrm{X}$-Rays and PC tomography to make a brilliant image of the body. It shows detailed pictures of body parts like bones, muscles, fat, and organs.

- Bronchoscopy: This is the test of the main airways of the lungs using a tube. It is utilized to assess and analyze lung issues, assess blockages, and take out a sample of tissue.

- Pleural fluid culture: In this test, from space among the lungs and the chest wall an example of liquid is taken $\mathrm{A}$ long, flimsy needle is put between the ribs and in the middle of the lungs and the chest wall. The Liquid is taken to the needle. It is shipped off the lab where it is tested to find out pneumonia.

- Ultrasound of the chest: In this an ultrasound is utilized to decide how much liquid is available and can help in deciding the cause of the liquid

- MRI: MRI is not commonly used to recognize pneumonia. MRI may give data about the irregularities like lung anomalies in light of abundance liquid, contamination, or the tumor.

- Needle Biopsy of the lung: To decide the reason for pneumonia Biopsy of the lung is finished by the specialist. Biopsies should be possible utilizing $\mathrm{x}$-beam, CT, ultrasound as well as MRI.

\section{COMPLICATIONS}

- Acute respiratory distress syndrome (ARDS): It is a type of disappointment in the respiratory.

- Lung abscess: In this, a pocket of discharge is a structure inside or around the lung. This discharge should be depleted out with a medical procedure.

- Respiratory disappointment: In this patient require a breathing machine.

- Sepsis: This is an infection in the blood that may lead to organ failure.

\section{TREATMENTS OF PNEUMONIA}

Some of the image treatments that may be used are as follows:

- Thoracentesis: In this therapy, fluid is taken out from the chest and look at by the specialist to confirm what germ is causing infection. X-beam, CT, and additionally ultrasound are finished during thoracentesis. The fluid eliminated during thoracentesis may likewise assist with giving alleviation.

- Chest tube placement: In this process, a meager plastic tube is inserted in the area between the chest wall and lungs. The tube can eliminate the abundance of liquid or air. This can be performed with the assistance of a CT scan or ultrasound.

- Image-guided abscess drainage: In this, with the help of an image we can directly place a needle in the opening of the abscess and can help during the installation of the drainage tube. When the abscess has framed, it tends to be eliminated by inserting a little seepage tube (pipe). Picture direction utilized fluoroscopy, x-ray, ultrasound or CT, is utilized.

\section{PROCEDURE FOR DIAGNOSIS OF PNEUMONIA}

This part clarifies, the interaction how of deep learning guidance is disclosed to distinguish pneumonia in clinical imaging. There are generally a few stages to diagnosing pneumonia specifically image processing, training, and classification. It is feasible to set/picture a model by using deep learning, that can separate between pictures from the marks of their classifications. Along these lines, utilizing top to bottom investigation of the finding of pneumonia, first, we need to gather pictures of the unhealthy lungs that will be secluded. The subsequent advance is to instruct the neural network until it recognizes contaminations. Finally independent new photographs. Here, new pictures that the model couldn't already see are shown in the model, and the model discovers the classification of those pictures.

\subsection{Image Acquisition Phase}

First, we have to gather pictures. To create a classification model, the PC can learn as a visual cue. The computer looks at multiple images to see the object. Yet, this paper talked about, the applicable subtleties expected to analyze pneumonia. Imaging pictures incorporate chest X-Ray, histopathology picture, sputum smear microscopy, CT scan. After this progression, the pictures will be utilized to prepare the model.

\subsection{Preprocessing Phase}

Doing advance is the second. In this progression, the image can be enhanced to improve the nature of the image. Image enhancement, for example, pulmonary embolism and bone misfortune can be utilized to identify Pneumonia. Data Augmentation can be used to expand the number of images. Feature identification can be applied, by this feature 
identification the deep learning model can identify the key factors to detect pneumonia. This progression is an assortment of images where the image quality is improved, or undesirable things are eliminated, for example, messes within photographs, commotion, Filtering. The release of this step is a modified image that will be used in training later.

\subsection{Training Phase}

In this stage, three components can be thought of. These features are the choice of deep learning algorithm, like the use of transfer learning and the use of an ensemble. There are numerous Deep learning methods, for instance, the deep belief network, the multilayer perceptron neural network, the recurrent neural network, and CNN referenced previously. Various algorithms have various styles of learning. Various kinds of data work better with various algorithms. CNN works splendidly with pictures. An algorithm should be picked dependent on the data in hand. Transfer of learning implies the exchange of information starting with one structure then onto the next. Ensemble refers to the utilization of more than one model during a split. Transfer learning and reconciliation procedures are utilized to reduce training time, improve class precision and decrease abuse. With the arrival of this progression, models are created from the data read.

\subsection{Classification Phase}

In this progression, the partition, the trained model ought to anticipate which class the image is in. If the model was prepared to disengage chest X-Ray pictures of solid lungs and Pneumonia lungs, it ought to have the option to appropriately recognize new (pictures that can not be seen/used earlier) from healthy lungs or lungs tainted with pneumonia. The model will focuses on picture procurement. The Opportunity score demonstrates that the picture might be under a specific classification. Toward the finish of this progression, the images will be arranged on the likelihood rating given by the model.

\section{AN ANALYSIS OF THE PNEUMONIA DETECTION SYSTEM IN RECENT YEARS}

$\mathrm{X}$-ray images, CT images, sputum smear microscopy, and histopathology images This kind of image are utilized to analyze pneumonia yet $X$-Ray images are well known because of the accessibility, openness, and versatility of X-Ray equipment. The use of features released by CNN is expanding, and the utilization of different features and a combination of features created by CNN stay low. This is because CNN is used for the automatic removal of the feature, eliminating the requirement to perform the feature manually [31]. The other features could not be used because many recent works have shown the rise of the features released by CNN in diagnosing Pneumonia. The number of jobs using data additions has increased steadily over the years as it is noted that data additions have boosted the accuracy of categories. CNN was favored in the deep learning algorithm because $\mathrm{CNN}$ is powerful and can produce categorized accuracy. Various tried examinations show that CNN has performed superior [32]. Various advantages of using CNN are automatic feature removal and the utilization of transfer learning benefits. Over the long haul, more work has been done on transfer learning. In transfer learning, there is no compelling reason to utilize another model. Transfer learning allows the highlights to take in while being prepared from old work to advance work and builds precision.

\section{ISSUES AND FUTURE OF PNEUMONIA DETECTION}

The first release comes from managing a large size of the image; here many people who are researching this direction reduce the original size of an image to reduce the cost of computation. It is very uneconomical to train at the actual image size and it takes time to train a knotty model. The second problem is that the available databases are limited, and therefore the amount of training data available is also small and training in a deep neural network with data limits leads to overfitting. The third problem is the high concentration of failures when using ensemble techniques: requires various errors for a group of dividers to do the best. It is expected that the foundations of planning will work together to produce better segregation results. In the future, it should be used to improve the performance of Pneumonia diagnosis. In the future, we can use ensemble learning An ensemble learning with different features provide better results. Perform training of images by cloud computing. It can solve the problem of handling the vast size of the image data. In the future, we have to resolve the problem of the limited dataset we can resolve it by making datasets available so that research in this direction is increased. In deep learning, research is limited to supervised learning algorithms, we can also explore this research for other learning algorithms like unsupervised or semi-supervised learning algorithms. We can use different features so that most researchers use features extracted by CNN. However, many features are still yet to be explored So that we can get new information from new features.

\section{CONCLUSION}

A Over time, more work on diagnosing Pneumonia has been published. However, there was an absence of accessible exploration on the present status of exploration and application. This review is subsequently conveyed an overview to detect pneumonia.

Summarize the concepts and concentrate on the work involved in the diagnosis of pneumonia, an Analysis of the Pneumonia Detection System in the Recent Year, in light of recognized attributes. From the investigation of the work distribution, the usage of $\mathrm{CNN}$, and transfer learning is large. 
Agrima Jain et al., International Journal of Emerging Trends in Engineering Research, 9(7), July 2021, 890 - 895

The remaining subject for discussion and progress of pneumonia diagnosis using deep learning was subsequently developed and described.

To conclude, how Computer-aided diagnosis was employed at pneumonia detection. It is exceptionally important to guarantee that future examination will focus on the right track, therefore there is a need of performance improvement in pneumonia detection systems. The future bearing proposed could improve the efficiency and also increase in number of computer-assisted pneumonia detection techniques.

\section{REFERENCES}

1. Douarre, C.; Schielein, R.; Frindel, C.; Gerth, S.; Rousseau, D. Transfer learning from synthetic data applied to soil-root segmentation in x-ray tomography images. J. Imaging 2018, 4, 65. [CrossRef].

2. Zhang, Y.; Wang, G.; Li, M.; Han, S. Automated classification analysis of geological structures based on images data and deep learning model. Appl. Sci. 2018, 8, 2493. [CrossRef].

3. Wang, Y.; Wang, C.; Zhang, H. Ship classification in high-resolution SAR images using deep learning of small datasets. Sensors 2018, 18, 2929. [CrossRef] [PubMed].

4. Sun, C.; Yang, Y.; Wen, C.; Xie, K.; Wen, F. Voiceprint identification for limited dataset using the deep migration hybrid model based on transfer learning. Sensors 2018, 18, 2399. [CrossRef] [PubMed]

5. Chen, Z.; Zhang, Y.; Ouyang, C.; Zhang, F.; Ma, J. Automated landslides detection for mountain cities using multi-temporal remote sensing imagery. Sensors 2018, 18, 821. [CrossRef]

6. Razzak, M.I.; Naz, S.; Zaib, A. Deep learning for medical image processing: Overview, challenges and the future. In Classification in BioApps; Springer: Cham, Switzerland, 2018; pp. 323-350.

7. Esteva, A.; Kuprel, B.; Novoa, R.A.; Ko, J.; Swetter, S.M.; Blau, H.M.; Thrun, S. Dermatologist-level classification of skin cancer with deep neural networks. Nature 2017, 542, 115-118. [CrossRef]

8. Milletari, F.; Navab, N.; Ahmadi, S.A. V-net: Fully convolutional neural networks for volumetric medical image segmentation. In Proceedings of the International Conference on 3D Vision, Stanford, CA, USA, 25-28 October 2016; pp. 565-571.

9. Gulshan, V.; Peng, L.; Coram, M.; Stumpe, M.C.; Wu, D.; Narayanaswamy, A.; Venugopalan, S.; Widner, K.; Madams, T.; Cuadros, J.; et al. Development and validation of a deep learning algorithm for detection of diabetic retinopathy in retinal fundus photographs. JAMA 2016, 316, 2402-2410. [CrossRef]

10. Grewal, M.; Srivastava, M.M.; Kumar, P.; Varadarajan, S. Radnet: Radiologist level accuracy using deep learning for hemorrhage detection in ct scans. In Proceedings of the 2018 IEEE 15th International Symposium on
Biomedical Imaging, Washington, DC, USA, 4-7 April 2018; pp. 281-284.

11. Bar, Y.; Diamant, I.; Wolf, L.; Lieberman, S.; Konen, E.; Greenspan, H. Chest pathology detection using deep learningwithnon-medicaltraining.

InProceedingsofthe2015IEEE12thInternationalSymposi umon Biomedical Imaging (ISBI), New York, NY, USA, 16-19 April 2015; pp. 294-297.

12. Nasrullah, N.; Sang, J.; Alam, M.S.; Mateen, M.; Cai, B.; Hu, H. Automated Lung Nodule Detection and Classification Using Deep Learning Combined with Multiple Strategies. Sensors 2019, 19, 3722. [CrossRef] [PubMed]

13. Yao, L.; Poblenz, E.; Dagunts, D.; Covington, B.; Bernard, D.; Lyman, K. Learning to diagnose from scratch by exploiting dependencies among labels. arXiv 2017, arXiv:1710.10501.

14. Khatri, A.A.R.J.; Vashista, H.; Mittal, N.; Ranjan, P.; Janardhanan, R. Pneumonia Identification in Chest $\mathrm{X}$-Ray Images Using EMD. In Trends in Communication, Cloud, and Big Data; Springer: Singapore, 2020; pp. 87-98.

15. Abiyev, R.H.; Ma'aitah, M.K.S. Deep convolutional neural networks for chest diseases detection. J. Healthc. Eng. 2018, 2018, 4168538. [CrossRef] [PubMed]

16. Stephen, O.; Sain, M.; Maduh, U.J.; Jeong, D.U. An efficient deep learning approach to pneumonia classification in healthcare. J. Healthc. Eng. 2019, 2019, 4180949. [CrossRef] [PubMed]

17. Cohen, J.P.; Bertin, P.; Frappier, V. Chester: A Web Delivered Locally Computed Chest X-Ray Disease Prediction System. arXiv 2019, arXiv:1901.11210.

18. Rajaraman, S.; Candemir, S.; Kim, I.; Thoma, G.; Antani, S. Visualization and interpretation of convolutional neural network predictions in detecting pneumonia in pediatric chest radiographs. Appl. Sci. 2018, 8, 1715. [CrossRef]

19. Sirazitdinov, I.; Kholiavchenko, M.; Mustafaev, T.; Yixuan, Y.; Kuleev, R.; Ibragimov, B. Deep neural network ensemble for pneumonia localization from a large-scale chest x-ray database. Comput. Electr. Eng. 2019, 78, 388-399. [CrossRef]

20. Lakhani, P.; Sundaram, B. Deep learning at chest radiography: Automated classification of pulmonary tuberculosis by using convolutional neural networks. Radiology 2017, 284, 574-582. [CrossRef]

21. Rajpurkar, P.; Irvin, J.; Ball, R.L.; Zhu, K.; Yang, B.; Mehta, H.; Duan, T. Deep learning for chest radiograph diagnosis: A retrospective comparison of the $\mathrm{CheXNeXt}$ algorithm to practicing radiologists. PLoS Med. 2018, 15, e1002686. [CrossRef]

22. Saraiva, A.; Santos, D.; Costa, N.J.C.; Sousa, J.V.M.; Ferreira, N.F.; Valente, A.; Soares, S. Models of Learning to Classify X-ray Images for the Detection of Pneumonia using Neural Networks. 2019. Available online: https://www.semanticscholar.org/paper/Models-of-Lear 
Agrima Jain et al., International Journal of Emerging Trends in Engineering Research, 9(7), July 2021, 890 - 895

ning-to-Classify-X-ray-Images-

for-the-Saraiva-Santos/0b8f202505b3d49c42fd45d86ec

a5dbd0b76fded?p2df (accessed on 18 June 2020).

23. Ayan, E.; Ünver, H.M. Diagnosis of Pneumonia from Chest X-Ray Images Using Deep Learning. In Proceedings of the 2019 Scientific Meeting on Electrical-Electronics and Biomedical Engineering and Computer Science (EBBT), Istanbul, Turkey, 2-26 April 2019; pp. 1-5.

24. Rahman, T.; Chowdhury, M.E.; Khandakar, A.; Islam, K.R.; Islam, K.F.; Mahbub, Z.B.; Kadir, M.A.; Kashem, S. Transfer Learning with Deep Convolutional Neural Network (CNN) for Pneumonia Detection using Chest X-ray. Appl. Sci. 2020, 10, 3233. [CrossRef]

25. Xiao, Z.; Du, N.; Geng, L.; Zhang, F.; Wu, J.; Liu, Y. Multi-scale heterogeneous 3D CNN for false-positive reduction in pulmonary nodule detection, based on chest CT images. Appl. Sci. 2019, 9, 3261. [CrossRef]

26. Xu, S.; Wu, H.; Bie, R. CXNet-m1: Anomaly detection on chest X-rays with image based deep learning. IEEE Access 2018, 7, 4466-4477. [CrossRef]

27. Jaiswal, A.K.; Tiwari, P.; Kumar, S.; Gupta, D.; Khanna, A.; Rodrigues, J.J. Identifying pneumonia in chest X-rays: A deep learning approach. Measurement 2019, 145, 511-518. [CrossRef]

28. Jung, H.; Kim, B.; Lee, I. Classification of lung nodules in CT scans using three-dimensional deep convolutional neural networks with a checkpoint ensemble method. BMC Med. Imaging 2018, 18, 48. [CrossRef]

29. Chouhan, V.; Singh, S.K.; Khamparia, A.; Gupta, D.; Tiwari, P.; Moreira, C.; Damaševičius, R.; de Albuquerque, V.H.C. A Novel Transfer Learning Based Approach for Pneumonia Detection in Chest X-ray Images. Appl. Sci. 2020, 10, 559. [CrossRef]

30. Mohd. Farukh Hashmi, Satyarth katihar, Avinash G Keskar, Neeraj Dhanraj Bokde and Zong Woo Geem. Efficient Pneumonia Detection in Chest Xray Images Using Deep Transfer Learning. Received: 17 May 2020; Accepted: 16 June 2020; Published: 19 June 2020.

31. Al-Ajlan, A.; Allali, A.E. CNN-MGP: Convolutional Neural Networks for Metagenomics Gene Prediction. Interdiscip. Sci. Comput. Life Sci. 2019, 11, 628-635. [CrossRef] [PubMed]

32. Abbas, A.; Abdelsamea, M.M. Learning Transformations for Automated Classification of Manifestation of Tuberculosis using Convolutional Neural Network. In Proceedings of the 2018 13th International Conference on Computer Engineering andSystems (ICCES), Cairo, Egypt, 18-19 December 2018; IEEE: New York, NY, USA, 2018; pp. 122-126.

33. Stefanus Tao Hwa Kieu 1, Abdullah Bade 1, Mohd Hanafi Ahmad Hijazi 2,* and Hoshang Kolivand 3 A Survey of Deep Learning for Lung Disease Detection on Medical Images: State-of-the-Art, Taxonomy, Issues and Future Directions. Received: 24 October 2020; Accepted: 25 November 2020; Published: 1 December 2020.
34. Sabri, N., Hamzah, R., Ibrahim, S., \& Abu Samah, K. A. F. (2020). COVID-19 detection for chest X-ray images using local binary pattern. International Journal of Emerging Trends in Engineering Research, $8(1$ Special Issue 1) https://doi.org/10.30534/ijeter/2020/1281.12020 